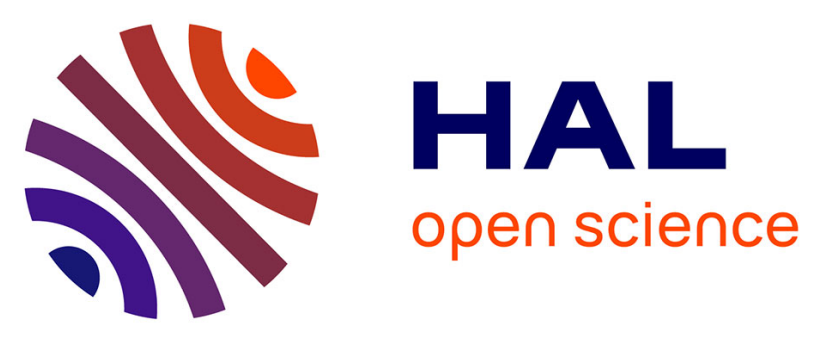

\title{
Zero degree Cherenkov calorimeters for the ALICE experiment
}

\author{
A. de Falco, R. Arnaldi, E. Chiavassa, C. Cicaló, P. Cortese, N. de Marco, G. \\ Dellacasa, A. Ferretti, M. Floris, M. Gagliardi, et al.
}

\section{- To cite this version:}

A. de Falco, R. Arnaldi, E. Chiavassa, C. Cicaló, P. Cortese, et al.. Zero degree Cherenkov calorimeters for the ALICE experiment. RICH 2007 - Sixth International Workshop on Ring Imaging Cherenkov Detectors, Oct 2007, Trieste, Italy. pp.267-269, 10.1016/j.nima.2008.07.070 . in2p3-00343634

\section{HAL Id: in2p3-00343634 https://hal.in2p3.fr/in2p3-00343634}

Submitted on 2 Dec 2008

HAL is a multi-disciplinary open access archive for the deposit and dissemination of scientific research documents, whether they are published or not. The documents may come from teaching and research institutions in France or abroad, or from public or private research centers.
L'archive ouverte pluridisciplinaire HAL, est destinée au dépôt et à la diffusion de documents scientifiques de niveau recherche, publiés ou non, émanant des établissements d'enseignement et de recherche français ou étrangers, des laboratoires publics ou privés. 


\title{
Zero Degree Cherenkov calorimeters for the ALICE experiment
}

\author{
A. De Falco ${ }^{a, b}$ R. Arnaldi ${ }^{c}$ E. Chiavassa ${ }^{c, d}$ C. Cicaló ${ }^{b}$ \\ P. Cortese ${ }^{c, e}$ G. Dellacasa ${ }^{c, e}$ A. Ferretti ${ }^{c, d}$ M. Floris ${ }^{a, b}$ \\ M. Gagliardi ${ }^{c, d}$ M. Gallio ${ }^{c, d}$ R. Gemme ${ }^{c, e}$ A. Masoni ${ }^{b}$ \\ P. Mereu ${ }^{c}$ A. Musso ${ }^{c}$ C. Oppedisano ${ }^{c}$ A. Piccotti ${ }^{c}$ F. Poggio ${ }^{c, d}$ \\ G. Puddu ${ }^{a, b}$ E. Scomparin ${ }^{c}$ S. Serci ${ }^{a, b}$ E. Siddi ${ }^{a, b}$ D. Stocco ${ }^{c, d}$ \\ G. Usai ${ }^{a, b}$ E. Vercellin ${ }^{c, d}$ F. Yermia ${ }^{f}$ \\ for the ALICE Collaboration \\ ${ }^{a}$ Università di Cagliari, Italy \\ ${ }^{\mathrm{b}}$ INFN Sezione di Cagliari, Italy \\ ${ }^{\mathrm{c}}$ INFN Sezione di Torino, Italy \\ ${ }^{\mathrm{d}}$ Università di Torino, Italy \\ ${ }^{\mathrm{e}}$ Università del Piemonte Orientale, Italy \\ ${ }^{\mathrm{f}}$ Laboratoire SUBATECH, Nantes, France
}

\begin{abstract}
The collision centrality in the ALICE experiment will be determined by the Zero Degree Calorimeters (ZDCs), that will measure the spectator nucleons energy in heavy ion collisions. The ZDCs detect the Cherenkov light produced by the fast particles in the shower that cross the quartz fibers, acting as the active material embedded in a dense absorber matrix. Test beam results of the calorimeters are presented.
\end{abstract}

The ALICE experiment [1] will study the properties of matter at extreme temperature and energy density conditions produced in $\mathrm{Pb}-\mathrm{Pb}$ collisions at $\sqrt{s}=5.5 A \cdot \mathrm{TeV}$, in the search for the Quark-Gluon Plasma (QGP) phase, using the LHC facility at CERN. The most favorable conditions for the QGP formation will be obtained in central collisions. The collision impact parameter can be estimated by means of the Zero Degree Calorimeters (ZDC), that detect the energy carried by the non-interacting (spectator) nucleons which are unaffected by the collision and fly at $0^{\circ}$ with respect to the beam direction [2]. Protons and neutrons will be separated by the LHC separator magnet: the former will be detected by a proton calorimeter (ZP) internal to the LHC ring, the latter by a neutron calorimeter $(\mathrm{ZN})$ located between the two beam 
pipes. Two sets of calorimeters will be placed on both sides at about $116 \mathrm{~m}$ from the intersection point (IP). Two electromagnetic calorimeters (ZEM), located at $7.5 \mathrm{~m}$ from the IP will complement the ZDC information, providing the electromagnetic energy produced in the forward direction. They cover the pseudorapidity range $4.8<\eta<5.7$.

In order to operate in the ALICE environment, the ZDCs must provide a fast response for trigger purposes, be radiation tolerant (the deposited dose in the neutron calorimeter is expected to be $1 \mathrm{Mrad} /$ day at $L=10^{27} \mathrm{~cm}^{-2} \mathrm{~s}^{-1}$ ) and compact, due to the small amount of space allocated for the $\mathrm{ZN}$, and to the limited separation between the spectator protons spot and the beam pipe. These conditions can be fulfilled using quartz fibers as active material, that detect the Cherenkov light produced by shower's charged particles [3]. The emission process is intrinsically fast. The signal width from the detector is of the order of ten nanoseconds, making the detector suitable for trigger purposes. Quartz fibers calorimeters have already been proved to be radiation resistant, having been successfully used in the NA50 experiment [4], where they were exposed to a radiation level 10 times higher than that expected for 10 years operation in ALICE. Furthermore, due to the presence of a threshold in the Cherenkov effect, the device has a low sensitivity to the induced radioactivation. Finally, since the calorimeters detect the fast particles in the shower, mainly electrons and positrons, they are sensitive to the electromagnetic core of the hadronic shower, and therefore the transverse dimensions needed to contain the shower are limited. The ZN and ZP dimensions are $7.2 \cdot 7.2 \cdot 100 \mathrm{~cm}^{3}$ and $22.8 \cdot 12 \cdot 150 \mathrm{~cm}^{3}$ respectively.

The quartz fibers in the ZDCs are parallel to the beam axis, embedded in a dense absorber matrix composed of grooved slabs, stacked to form a parallelepiped. The fiber spacing, fiber diameter and filling ratio are $1.6 \mathrm{~mm}$, $365 \mu \mathrm{m}$ and $1 / 22$ for $\mathrm{ZN} ; 4 \mathrm{~mm}, 550 \mu \mathrm{m}$ and 1/65 for ZP. In order to reduce the shower dimensions, high density absorber materials were chosen: brass for $\mathrm{ZP}$ and a tungsten alloy for ZN $\left(8.48 \mathrm{~g} / \mathrm{cm}^{3}\right.$ and $17.6 \mathrm{~g} / \mathrm{cm}^{3}$ respectively).

The fibers emerge on the rear side of the calorimeter and are read by 5 Hamamatsu R329-02 photomultipliers. The ZDC readout is segmented into 4 towers. One out of two fibres is sent to a single photomultiplier (PMTc), while the remaining ones are connected to four different photodetectors (PMT1 to PMT4), which collect the light from each tower.

The calorimeters were tested at the CERN SPS using electron/positron and hadron beams with energy ranging from 50 to $200 \mathrm{GeV}$. ZN was also tested with an ${ }^{115} \mathrm{In}$ beam at $158 \mathrm{~A} \cdot \mathrm{GeV}$. The calorimeters were placed on a movable platform. A set of plastic scintillators provided the trigger, while the beam impact position on the calorimeter front face was measured by a MWPC. Muons were identified by means of two plastic scintillators placed behind an iron wall.

Fig. 1 shows the ADC spectrum for a $120 \mathrm{GeV}$ pion beam impinging on ZP. The spectrum for pions is strongly asymmetric, due to the large fluctuations in the electromagnetic component of the hadronic shower, caused 


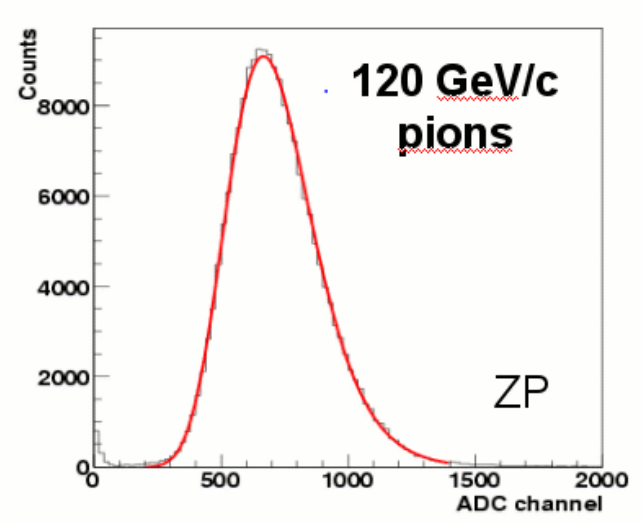

Fig. 1. ADC spectrum for a $120 \mathrm{GeV}$ pion beam on ZN

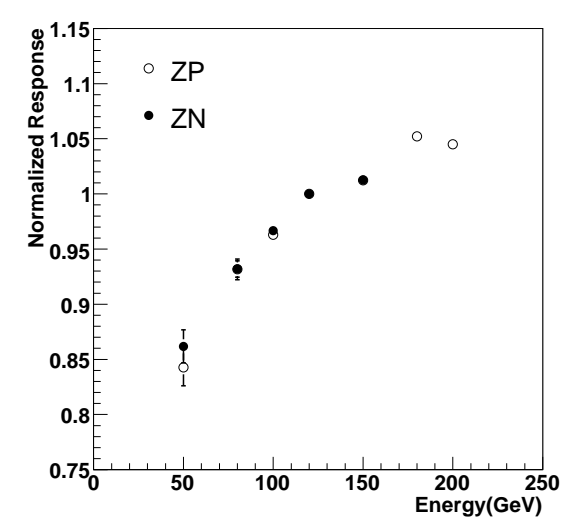

Fig. 2. Ratio between the response to hadrons and the beam energy normalized to the $120 \mathrm{GeV}$ value.

by the leading $\pi^{0}$ produced by charge exchange mechanism. To account for the asymmetric shape, hadron spectra were fitted with the function $f(x)=$ $N \exp \left(-(x-\mu)^{2} / 2 \sigma^{2}\right)$, where $\sigma=\sigma_{0}+\sigma_{1}(x-\mu) / \mu$.

The ratio between the ZDC response to hadron beams and the beam energy is plotted in fig. 2 as a function of the beam energy. The experimental points are normalized to the value at $120 \mathrm{GeV}$. A clear deviation from linearity is evident, and is due to the fact that the ZDCs are non-compensating devices. The ratio $\mathrm{e} / \mathrm{h}$, calculated from the measured $\mathrm{e} / \pi$ corrected for the lateral leakage, is $>5$ for $\mathrm{ZN}$ and $\sim 3$ for ZP. However, the non-linearity of the calorimeter response as a function of the energy is not a major concern, since the ZDCs will detect the number of spectator nucleons, each of them having roughly the same energy $(2.76 \mathrm{TeV})$.

The resolution as a function of the energy (fig. 3) was fitted with the usual formula $\sigma / E=a / \sqrt{(} E) \oplus b$, giving for hadron beams $a=(257 \pm 3) \% \mathrm{GeV}^{1 / 2}$, $b=(10.3 \pm 0.6) \%$ for $\mathrm{ZN}$ and $a=(237 \pm 2) \% \mathrm{GeV}^{1 / 2}, b=(12.5 \pm 0.2) \%$

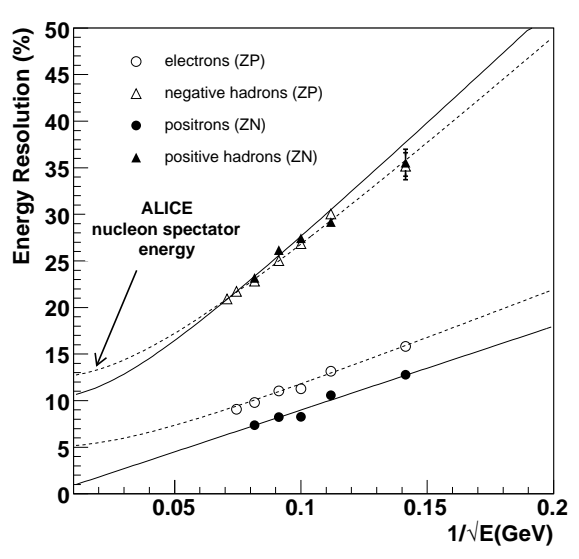

Fig. 3. Energy resolution for hadron and electron/positron beams.

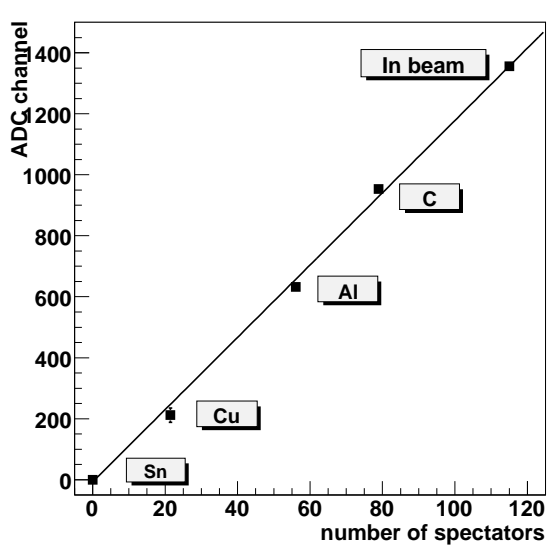

Fig. 4. ZN response as a function of the number of spectators. 
for ZP. The energy resolution extrapolated at $2.7 \mathrm{TeV}$, the spectator nucleons energy for $\mathrm{Pb}-\mathrm{Pb}$ collisions at the $\mathrm{LHC}$, is $11.4 \%$ and $13 \%$ for $\mathrm{ZN}$ and $\mathrm{ZP}$ respectively, of the order of the spectator energy fluctuations.

In order to check the uniformity of the response as a function of the beam impact point, a thin horizontal slice along the fibers plane was selected using the MWPC, and the response was determined as a function of the $\mathrm{x}$ coordinate. The variation for hadron beams is smaller than $2.5 \%$, showing that the fiber spacing is adequate for hadronic calorimetry.

The shower transverse size was determined moving the calorimeter with respect to the beam along the horizontal coordinate, and measuring the response of a single tower. Its derivative gives the shower's transverse profile, whose FWHM for hadron beams is $8 \mathrm{~mm}$ for ZN and $13 \mathrm{~mm}$ for ZP.

The neutron calorimeter can be used to detect the beam impact position on its front face according to the formula $x=\sum_{i=1}^{4} x_{i}^{q} A D C_{i}^{\alpha} / \sum_{i=1}^{4} A D C_{i}^{\alpha}$ (and similar for $\mathrm{y}$ ), where $x_{i}^{q}$ is the $\mathrm{x}$ position of of the $\mathrm{i}$-th tower center, $A D C_{i}$ the corresponding ADC response, and $\alpha=0.5$ is a parameter optimized with Monte Carlo simulations. The spatial resolution, determined comparing the calorimeter results to the measurement performed with the MWPC, is $\sim 3 \mathrm{~mm}$. It is thus possible to determine, event by event, the centroid of the spectator neutrons, in order to monitor the beam crossing angle at the interaction point and estimate the reaction plane of the nuclear collisions.

The neutron calorimeter was tested with an indium beam at an energy of $158 \mathrm{GeV}$ per nucleon, incident on several nuclear targets. Central collisions were selected looking at the low energy end point. The number of spectators $N_{\text {spec }}$ was determined under the geometric approximation, treating the nuclei as rigid spheres with uniform nucleon distribution. The energy deposited in

the ZDC can be expressed as $E_{Z D C}^{\text {endpoint }}=N_{\text {spec }} E_{\text {spec }}+N_{\text {part }} E_{\text {part }}$, where $E_{\text {part }}$ can be evaluated considering that in most central ${ }^{115} \mathrm{In}-{ }^{119} \mathrm{Sn}$ collisions, $N_{\text {spec }}=0, N_{\text {part }}=115$. This allows to correct the result for the energy deposited in the calorimeter by the participants. The response as a function of $N_{\text {spec }}$ is linear, as shown in fig. 4 . The energy resolution for the indium beam was also determined, and its value is $\sigma / E=2.8 \%$.

To conclude, the main characteristics of the ZDCs (linearity of the response as a function of the number of spectators, uniformity of the response versus the beam impact position, energy resolution, shower transverse dimensions) show that they fulfill the requirements for their use in ALICE.

\section{References}

[1] "ALICE Technical Proposal" CERN/LHCC 95-71

[2] Zero Degree Calorimeter Technical Design Report, CERN/LHCC, 1999.

[3] P.Gorodetzky et al., Nucl. Instr. Meth. A 361 (1995) 161.

[4] R. Arnaldi et al., Nucl. Instr. Meth. A 411 (1998) 1. 